\title{
WKB thresholds of standard, helical, and azimuthal magnetorotational instability
}

\author{
Oleg Kirillov and Frank Stefani
}

\author{
Helmholtz-Zentrum Dresden-Rossendorf POB 5101 19, 01314 Dresden, Germany \\ email: o.kirillov@hzdr.de, f.stefani@hzdr.de
}

\begin{abstract}
We consider rotating flows of an electrically conducting, viscous and resistive fluid in an external magnetic field with arbitrary combinations of axial and azimuthal components. Within the short-wavelength approximation, the local stability of the flow is studied with respect to perturbations of arbitrary azimuthal wavenumbers. In the limit of vanishing magnetic Prandtl number $(\mathrm{Pm})$ we find that the maximum critical Rossby number (Ro) for the occurrence of the magnetorotational instability (MRI) is universally governed by the Liu limit $\operatorname{Ro}_{L i u}=2-2 \sqrt{2} \approx$ -0.828 which is below the value for Keplerian rotation $\mathrm{Ro}_{K}$ epler $=-0.75$.
\end{abstract}

Keywords. Magnetorotational instability, WKB approximation, accretion disks

\section{Magnetorotational instability at small magnetic Prandtl numbers}

Nowadays, the magnetorotational instability (MRI) is widely accepted as the main source of turbulence and outward angular momentum transport in accretion disks (Balbus and Hawley, 1991). Recently, the influence of viscosity and electrical resistivity on the MRI has attracted particular interest (Pessah and Chan, 2008). The most important parameter in this respect is the magnetic Prandtl number, i.e. the ratio of viscosity to magnetic diffusivity. For accretion disks around black holes (BH), Balbus and Henri (2008) had discussed the transition from large values of Pm, in the vicinity of the $\mathrm{BH}$, to small values in the outer part of the disk. Invoking a thermal runaway process at the unstable interface between regions with $\mathrm{Pm}>1$ and $\mathrm{Pm}<1$, the authors associated this boundary with the existence of high and low X-ray states. Small values of Pm play also a role in protoplanetary disks, in particular in the so-called "dead zones", characterized by low degrees of ionization.

Besides this relevance to accretion disks, the limit of vanishing Pm has acquired some additional interest in connection with the recent liquid metal MRI experiments. While experiments on the standard MRI (SMRI), with only an axial magnetic field being applied (Sisan et al., 2004, Nornberg et al., 2010), require certain critical magnetic Reynolds and Lundquist numbers to succeed, experiments on the so-called helical MRI (HMRI) require only some critical Reynolds and Hartmann numbers and are, therefore, much easier implementable in the liquid metal lab (Hollerbach and Rüdiger, 2005).

However, the role of HMRI in astrophysics is still under debate. As early shown by Liu et al. (2006), the working of HMRI in the inductionless limit $\mathrm{Pm} \rightarrow 0$ is restricted to comparably steep rotation profiles with Rossby numbers $\operatorname{Ro}_{2}<\operatorname{Ro}_{L i u}=2(1-\sqrt{2}) \approx$ -0.8284 , and does therefore not extend to Keplerian profiles with $\operatorname{Ro}_{\text {Kepler }}=-0.75$. This essential limitation of the HMRI, together with a variety of further parameter dependencies, was confirmed in the PROMISE experiment (Stefani et al., 2006, Stefani et al., 2009). Only recently, the intricate, though continuous, transition between SMRI and HMRI was clarified by Kirillov and Stefani (2010). It also turned out that at small 
but finite Pm there exists an analogue of the Liu limit for HMRI that is just slightly closer to the Keplerian value: $\mathrm{Ro}_{c} \approx-0.802$, see Kirillov and Stefani (2011).

Interestingly, for the case of a purely or strongly dominant azimuthal magnetic field, Hollerbach et al. (2010) had predicted the occurrence of a non-axisymmetric version of MRI (azimuthal MRI, or AMRI), working apparently in a similar parameter region as HMRI. In a recent work, Kirillov et al. (2012) obtained the following explicit expression for the maximum critical Rossby number in the inductionless limit:

$$
\operatorname{Ro}_{c r}(\beta, n)=\frac{4 \beta^{4}+(\beta n+1)^{4}-\left(2 \beta^{2}+(\beta n+1)^{2}\right) \sqrt{4 \beta^{4}+(\beta n+1)^{4}}}{2 \beta^{2}(\beta n+1)^{2}} .
$$

Here, $\beta$ is proportional to the ratio of the azimuthal component to the axial one, and $n$ has the meaning of a re-scaled azimuthal wavenumber $(m)$ of the perturbation. The function $\operatorname{Ro}_{c r}(\beta, n)$ has its maximum exactly at the Liu limit when $\beta(n)=( \pm \sqrt{2}-n)^{-1}$, i.e. even in the case of AMRI corresponding to $\beta \rightarrow \infty$. Quite surprisingly, the above equation comprises not only HMRI $(m=0)$ and AMRI $(m=1)$, but also higher azimuthal modes $(m>1)$ that should become observable for comparably small values of $\beta$.

\section{Astrophysical implications}

Despite some ongoing debate about the possible role of conducting boundaries (Rüdiger and Hollerbach, 2007, Priede, 2011), it seems that all those inductionless versions of MRI, like HMRI and AMRI, are not applicable to rotation profiles as shallow as the Keplerian one. However, with main focus on low Pm flows, Umurhan (2010) had discussed the possibility that the saturation of MRI in accretion disks could lead to modified flow structures within parts of steeper shear, sandwiched with parts of shallower shear. Similarly, for protoplanetary disks the simulations of Kato et al. (2009) had also shown the appearance of layers with increased shear. By virtue of a possible sudden onset within such segments of steepening shear, the inductionless versions of MRI could still play a certain role in real astrophysical problems.

\section{References}

Balbus, S. A. \& Hawley, J. F. 1991, ApJ 376, 214.

Balbus, S. A. \& Henri, P. 2008, ApJ 674, 408.

Hollerbach, R. \& Rüdiger, G. 2005, Phys. Rev. Lett. 95, 124501.

Hollerbach, R., Teeluck, V., \& Rüdiger, G. 2010, Phys. Rev. Lett. 104, 044502.

Kato, M. T., Nakamura, K., Tandokoro, R., Fujimoto, M., \& Ida, S. 2009, ApJ 691, 1697.

Kirillov, O. N. \& Stefani, F. 2010, ApJ 712, 52.

Kirillov, O. N. \& Stefani, F. 2011, Phys. Rev. E 84, 036304.

Kirillov, O. N., Stefani, F., \& Fukumoto, Y. 2012, ApJ 756, 83.

Liu, W., Goodman, J., Herron, I., \& Ji, H. T. 2006, Phys. Rev. E 74, 056302.

Nornberg, M. D., Ji, H., Schartman, E., Roach, E., \& Goodman, J. 2010, Phys. Rev. Lett. 104, 074501 .

Pessah, M. E. \& Chan, C. 2008, ApJ 684, 498.

Priede, J. 2011, Phys. Rev. E 84, 066314.

Rüdiger, G. \& Hollerbach, R. 2007, Phys. Rev. E 76, 068301.

Sisan, D. R. et al. 2004, Phys. Rev. Lett. 93, 114502.

Stefani, F. et al. 2006, Phys. Rev. Lett. 97, 184502.

Stefani, F. et al. 2009, Phys. Rev. E 80, 066303.

Umurhan, O. M. 2010, A\&A 513, A47. 